\title{
Fragment size and dynamics of EGFR-mutated tumor-derived DNA provide prognostic information regarding EGFR-TKI efficacy in patients with EGFR- mutated NSCLC
}

Kei Kunimasa ( $\nabla$ kei.kunimasa@oici.jp )

Osaka International Cancer Institute

Kazumi Nishino

Osaka International Cancer Institute

Yoshiharu Sato

DNA Chip Research Inc

Masahide Mori

Osaka Toneyama Medical Center

Shoichi Ihara

Osaka Police Hospital

Hidekazu Suzuki

Osaka Habikino Medical Center

Izumi Nagatomo

Osaka University

Toru Kumagai

Osaka International Cancer Institute

Fumio Imamura

Osaka International Cancer Institute

\section{Research Article}

Keywords: EGFR-mutated lung cancer, circulating tumor DNA, osimertinib, ctDNA fragmentation, nextgeneration sequencing

Posted Date: February 8th, 2022

DOI: https://doi.org/10.21203/rs.3.rs-1257298/v1

License: (9) (1) This work is licensed under a Creative Commons Attribution 4.0 International License.

Read Full License 


\section{Abstract}

Circulating tumor DNA (ctDNA)-based next-generation sequencing (NGS) is a complementary and alternative test to tissue-based NGS. e performed NGS analysis of ctDNA samples collected from patients with EGFR-mutated non-small cell lung cancer (NSCLC) who received osimertinib; the samples were collected after second-line treatment, before osimertinib treatment, one week and one month after osimertinib treatment, and at the time of resistance formation, and the correlation with osimertinib efficacy was examined. From January to December 2018, 34 patients with EGFR-mutated NSCLC harboring EGFRT790M mutations were enrolled, and a total of 132 peripheral blood samples were collected. The fragment sizes of EGFR-mutated ctDNAs were significantly shorter than those of their corresponding normal fragments. Osimertinib treatment of EGFR-mutated patients with shorter EGFRmutated ctDNA fragments resulted in shorter progression-free survival (PFS). Disappearance timing of EGFR-mutated fragment fractions and clonal evolution patterns (new driver mutation group, additional mutation group vs. attenuation group) were each associated with osimertinib PFS, although multivariate analysis revealed that only shorter EGFR-mutated ctDNA fragments were associated with osimertinib PFS. EGFR-mutated ctDNA fragment size, disappearance timing, and clonal evolution pattern were related to the effects of osimertinib. In particular, short EGFR-mutated ctDNA fragmentation may be closely related to osimertinib efficacy prediction.

\section{Introduction}

In solid tumors, including lung cancer, the guidelines recommend identification of driver gene mutations and treatment with the corresponding molecular targeted therapies when systemic chemotherapy is introduced, because of their efficacy $(1,2)$. In lung cancer, advancements have been made in the identification of druggable driver mutations, and detection of multiple druggable driver mutations before treatment is essential( 1,2$)$. To identify these mutations, next-generation sequencing (NGS) is being rapidly implemented in clinical practice(3). However, unlike other cancer types, lung cancer requires successful NGS with the relatively small specimens obtainable from bronchoscopic biopsies, especially in advanced cases, and it may not be possible to secure sufficient tumor tissue specimens for successful NGS analysis(4). In such situations, liquid biopsy, in which NGS analysis is based on evaluation of cellfree DNA (cfDNA) or circulating tumor-derived DNA (ctDNA) using peripheral blood plasma, can be an extremely powerful tool in lung cancer treatment because it does not require tissue samples(5-7).

Research on liquid biopsy to detect driver mutations in primary tumors by analyzing ctDNA has been accelerating in recent years(7). NGS panel analysis based on ctDNA is expected to soon be implemented in clinical practice $(8,9)$. However, NGS analysis of ctDNA that is not seen in DNA from tissues may be associated with problems. ctDNA is fragmented at lower concentrations than tissues, so the variant allele frequency (VAF) detected tends to be lower, and the possibility of false-positive or false-negative results associated with this should be considered(10). To solve these problems, methods have been developed to increase the sensitivity of NGS analysis of ctDNA(11). Barcode sequences with lengths of 10-15 base pairs are added to each ctDNA fragment to distinguish the sequence read of each fragment $(12,13)$. 
However, the disadvantage of this method is that errors in the barcode sequence affect the sensitivity of the technique(11), since the final sequence reads do not represent the original ctDNA population due to the global amplification step during template preparation. To overcome this problem, small collections of barcode sequences have been designed to detect and exclude erroneous sequences. However, this approach requires each barcode sequence to be individually manufactured, and it cannot accommodate large numbers of sequences.

To achieve accurate ctDNA NGS analysis, we recently established a high-fidelity target-sequencing system of individual molecules identified in ctDNA by using barcode sequences, which was named the non-overlapping integrated reads (NOIR) sequencing system $(11,14)$. This system consists of the following steps. First, a novel target sequencing method is used to add barcode sequences via adapter ligation. This method uses linear amplification to eliminate errors introduced in the barcode sequence during the early cycles of PCR. Second, erroneous barcode tags are monitored and removed(11). This process involves the identification of individual molecules that have been sequenced and have undergone absolute quantitation of the number of mutations. Using this method, we succeeded in evaluating the response to anticancer drugs by sequencing ctDNA in patients with advanced pancreatic cancer(15). In the present study, we analyzed ctDNA by using the NOIR sequencing system in patients with non-small cell lung cancer (NSCLC) harboring EGFR T790M mutation to evaluate the therapeutic effect and behavior of tumor subclones during osimertinib treatment.

\section{Results}

\section{Patient characteristics}

From January to December 2018, 34 patients with EGFR-mutated NSCLC harboring the EGFR T790M mutation acquired after the first EGFR-TKI treatment were treated with osimertinib monotherapy. The clinical characteristics of the patients are summarized in Table 1. The median age of these patients was 66 years (IQR, 39-83 years); 27 patients (79.4\%) were female; 22 patients $(64.7 \%)$ were never smokers,

and 17 patients $(50 \%)$ had brain metastases after osimertinib administration. The EGFR mutation profiles were as follows: Ex.19 del (58.8\%), Ex.21 L858R (41.2\%), and resistance mutation T790M (100.0\%). Ten patients $(29.4 \%)$ received osimertinib as second-line treatment, 11 (32.4\%) received it as third-line treatment, and 13 patients (38.2\%) received it after the fourth line of treatment. Median PFS with osimertinib treatment was 6.6 months (95\% confidence interval [Cl]: 5.1-12.9) in all patients, 7.1 months (95\% Cl: 5.1-15.3) in patients with the Ex.19 deletion, and 6.0 months (95\% Cl: 2.8-15.5) in those with the Ex.21 L858R mutation. There was no significant difference in median PFS among the major EGFR mutations.

\section{Fragmentation of EGFR-mutated ctDNA predicts osimertinib efficacy}

A total of 34 peripheral blood samples were collected from points $A$ to $C$, and 30 samples were collected from point D (Fig. 1A). Median cfDNA concentration ( $\mathrm{ng} / \mu \mathrm{L})$ at point A was $0.6 \mathrm{ng} / \mu \mathrm{L}(\mathrm{IQR}, 0.3-4.2 \mathrm{ng} /$ $\mu \mathrm{L})$, and the median total DNA content was $115 \mathrm{ng}$ (IQR, 67-836 ng). The median optical density (OD) 
260/280 of cfDNA was 1.79 (IQR, 1.63-1.92). The fragment sizes of ctDNA of Ex.19 del $(n=44)$, Ex.20 T790M ( $n=61)$, and Ex.21 L858R ( $n=38)$ were significantly smaller than those of their corresponding normal EGFR coding fragments (Fig. 1B). The mean size of each ctDNA fragment is shown in Figure 1C. Notably, osimertinib treatment of EGFR-mutated patients with a shorter EGFR-mutated ctDNA fragment (4.9 months; $95 \% \mathrm{Cl}: 2.8-13.8)$ resulted in a shorter PFS relative to that of patients with a longer EGFRmutated ctDNA fragment (11.0 months; $95 \% \mathrm{Cl}$ : 5.5-15.5; short vs. long; $\mathrm{P}=0.002)$.

\section{Timing of disappearance of EGFR-mutated ctDNA is not associated with osimertinib efficacy}

A positive correlation was found between Ex.19 del, L858R VAF, and T790M VAF at point A $(r=0.837)$ (Fig. 2A). In 19 cases (55.9\%) in which primary tissue specimens were obtained at point A, we examined whether the T790M location was cis or trans to Ex.19 del or L858R, and 18 (94.7\%) were found to have the T790M mutation in the cis position (Fig. 2B). In one case, the T790M mutation was in the trans position. All 34 cases were classified by the time of disappearance of both the Ex.19 or L858R and T790M mutation alleles. Those that showed disappearance at point $B$ were classified into the $A \rightarrow B$ disappearance group $(n=9)$; those that showed disappearance at point $C$ were classified into the $A \rightarrow C$ disappearance group $(n=15)$; and the remaining cases were classified into the no disappearance group $(n=10)$. The three groups showed no significant difference in the T790M VAF at point $A(P=0.379)$ (Fig. 2C) and no significant difference in PFS with osimertinib treatment $(P=0.442)$ (Fig. 2D).

\section{Evolution of the resistance mutational pattern is associated with osimertinib efficacy}

In the evaluations of the mutation profile at point $D, 30$ cases that could be analyzed were classified into the following three groups. The first was a group in which EGFR Ex.19, L858R, and T790M mutations disappeared and a new mutation appeared at oncogenic driver genes other than EGFR (new driver mutation group; $\mathrm{n}=13$ ); the second was a group in which a new oncogenic driver mutation was added to the existing EGFR mutation (additional mutation group; $\mathrm{n}=6$ ); and the third was a group in which only the existing EGFR mutations were detected (attenuation group; $\mathrm{n}=11$ ). Two representative examples of each of these three groups are shown in Fig. 3A. The additional mutation group showed a significantly shorter PFS (3.8 months; 95\% Cl: 0.9-NA) among the three groups $(P<0.05)$ (Fig. 3B).

\section{NGS concordance of tissue and plasma samples and co-mutation profile}

A total of 19 cases for which tissue samples at point A were available were analyzed using NGS with the NOIR-SS panel to assess the concordance rate with plasma samples. The results are shown as a mutation heat map in Fig. 4A. A total of 100 mutations were detected in the plasma samples and the corresponding tumor tissue at point A. Of the 100 mutations, $29(29 \%)$ were shared among both samples, $41(41 \%)$ were detected only in tissue, and 30 (30\%) were detected only in plasma (Fig. 4B). The mutations detected in 16/19 (84.2\%) cases were consistent only for EGFR major mutations (16). Based on the sequencing of plasma ctDNA at point A, 24 cases were classified as co-mutation-positive (Fig. 4C). No significant difference in PFS was found between the co-mutation-positive and-negative groups with osimertinib treatment $(P=0.08)$ (Fig. 4D). 


\section{Predictive ctDNA biomarker for osimertinib efficacy}

Multivariate analysis of the influence of ctDNA biomarkers on osimertinib efficacy for patients with EGFRmutation demonstrated that short fragmentation of EGFR-mutated ctDNA was an independent predictive factor for PFS in osimertinib treatment after second-line therapy (HR, 3.76; 95\% Cl: 1.17-12.1) $(P<0.05)$ (Table 2).

\section{Discussion}

The use of liquid biopsy, which mainly employs ctDNA analysis, is growing in many types of cancers, including NSCLC. Recent technological innovations in ctDNA analysis have enabled the utilization of ctDNA analysis in a way that is compatible with clinical practice, and two commercially available plasmabased NGS assays (Guardant360 and FoundationOne Liquid CDx) have received Food and Drug Administration approval. In addition to identifying druggable oncogenic mutations, ctDNA analysis has been widely used to identify new biomarkers, assess tumor clonal evolution, and obtain information for subsequent therapies. In this study, we demonstrated that ctDNA fragmentation is a significant predictor of the response to osimertinib therapy in EGFR-mutated NSCLC patients with the T790M mutation. Dynamic changes in EGFR-mutated ctDNA and clonal evolution may also be predictive of osimertinib efficacy, and further analysis of the clinical application of ctDNA is required.

Tumor-derived ctDNA is known to be fragmented and shorter than circulating cell-free DNA $(17,18)$. ctDNA fragmentation has been reported to be associated with cancer prognosis and malignancy $(15,19-21)$, and searching for fragmented ctDNA has also been suggested to be effective in detecting tumor-derived mutations $(22,23)$. Observations regarding differences in fragment length size and, to a lesser extent, periodicity could be translated to a patient with widely metastatic melanoma, where the principal peak associated with the BRAF V600E mutation was shorter than the wild-type distribution. Similar differences in fragment size were also observed in patients with lung cancer harboring common EGFR major activating mutations $(24,25)$. Tumor-derived ctDNA is believed to be released during apoptosis and necrosis of tumor cells $(26,27)$, and nucleosome-bound cancer DNA is more susceptible to endonucleases in the process, making it more likely to be fragmented(28). ctDNAs of $100 \mathrm{bp}$ or less are classified as ultrashort-fragment ctDNAs (29), and ctDNAs with hotpoint mutations are often classified in this category(25).. In the present study, the ctDNA size of the major mutations of EGFR, namely, T790M, Ex.19 del, and L858R, ranged from 90.77 to 94.96 bp in mean size (Fig. 1B, C). Fragmentation of ctDNA is related to the degree of tumor cell apoptosis in the tumor microenvironment or the degree of local inflammation in the tumor, and ultrashort ctDNA fragments may be surrogate markers of the degree of local tumor inflammation. EGFR-TKIs may be less effective when local tumor inflammation is severe(30, 31), which may account for the lower efficacy of osimertinib in patients with ultra-short fragments (Fig. 1D). Ultra-short fragmentation ctDNA cannot be detected by RT-PCR in terms of size(29), and accurate measurement of mutated ctDNA by the NOIR sequencing system(11) may have successfully identified biomarkers of fragmentation ctDNA. 
All enrolled patients in this study were treated with osimertinib after acquiring resistance mutations in EGFR T790M following EGFR-TKI therapy. In most cases, in which the positional relation between T790M and major activating mutations could be examined using tissue samples, it was located in the cis location, and the VAF for each mutation showed a strong correlation (Fig. 2A, B). It was expected that the mutant allele dynamics of T790M and the major activating mutations were identical. The dynamics of ctDNA, especially the disappearance of mutant alleles after the introduction of osimertinib, have been reported to predict long-term efficacy(32-34). In this study, we also observed a trend toward a longer response to osimertinib in patients showing early disappearance of T790M and major activating mutation alleles, although the difference was not significant.

Analysis of the mechanisms of osimertinib resistance in the phase 3 AURA3 trial (35), which compared osimertinib with platinum-pemetrexed combination chemotherapy in EGFRT790M-positive NSCLC patients following progression during prior EGFR-TKI treatment, showed that $21 \%$ of the patients acquired additional compound EGFR mutations and retained the T790M mutation even after they showed osimertinib resistance (36). In the present study, the C797S mutation, which is the most common tertiary EGFR mutation (35), was also detected in one patient and was found to be in the cis position relative to the T790M mutation (additional mutation type). In cases showing compound EGFR mutations, retention of the T790M mutation suggests that this additional EGFR mutation may be in the cis position. In approximately $50 \%$ of cases, the T790M mutation allele disappears in resistance, and the manifestation of new driver mutations, such as KRAS and MET amplification, can be recognized. Our study also found new driver mutations in 13/30 (43.3\%) patients (Fig. 3), which is consistent with these findings. Analysis of the resistance mechanism in the AURA 3 trial and the effect of osimertinib showed that loss of the T790M mutation allele was associated with a shorter PFS of osimertinib(37). In our study, the addition of a new driver mutation (additional mutation type) was associated with a shorter PFS, and the loss of the T790M mutation allele tended to result in a longer PFS than the additional mutation type. The following factors may account for these differences. First, in our study, 24/34 (70.6\%) patients received post-thirdline osimertinib therapy, and it is likely that the clonality in these tumors was different from that in the AURA 3 trial population due to the effects of drugs other than EGFR-TKIs. Second, the previous study only investigated the loss of the T790M mutation allele and did not estimate the behavior of major activating EGFR mutation alleles (37). Our results suggest that clones with an additional novel driver mutation in EGFR T790M and major activating mutations are resistant to osimertinib and may be related to shorter PFS.

The serial ctDNA analysis in our study showed the emergence of TP53 and ERBB2 mutations in the middle (point $B$ or $C$ ) and their disappearance in the resistant cases (point $D)$. Under treatment pressure, a new mutant clone may appear and disappear rapidly in the tumor(38). In the future, when evaluating the genomic profile of the primary tumors using ctDNA, the timing of blood collection and the timing of treatment change must also be considered. The timing of the appearance of new mutations, resistance formation in ctDNA, and tumor progression in images should be studied in conjunction with each other, and guidelines for utilizing ctDNA-guided treatment based on prospective studies should be developed in the future. 
In the present study, the concordance rates of tissue- and plasma-based NGS were as low as 29/70 (41.4\%) (Fig. 4A, B). In a previous report, the concordance rate of major druggable mutations between plasma- and tissue-based NGS using Guardant 360 was $77.6 \%(16)$. In this study, the concordance rate of EGFR major mutations was $84.2 \%$, but the concordance rate of TP53 mutations was especially low (Fig. 4A). This study included many cases after a relatively long period of treatment, and the heterogeneity of the primary and metastatic lesions at this point may have possibly increased. In addition, there have been no studies using the same analytical panel for tissue and plasma in previous reports, but this study used the same panel for both. CtDNA tends to be fragmented(23,39), and differences in the quality of DNA in comparison with the DNA extracted from tissue may have led to differences in detection rates. The concordance rate could be maintained in the case of major druggable mutations (16),, but the concordance rate might be lower in the case of other mutations. The effect of EGFR-TKIs on patients with EGFR-mutation is known to be influenced by co-mutations other than major EGFR mutations; however, this study failed to confirm the effect of co-mutations on the effect of EGFRTKIs (Fig. 4D). In this study, only a small number of patients were analyzed, and the timing of EGFR-TKI administration was inconsistent. More than $50 \%$ of the patients received late line EGFR-TKI administration (Table 1).

This study had the following limitations. Samples were collected for only 34 cases, and sample collection from points $A$ to $D$ was successfully performed only in 30 cases. In addition, tissue samples at point $A$ were successfully collected in only 19 cases, and successful comparisons with tissue samples were performed in only a small number of cases. The gene panel used in this analysis was a very small panel of only nine genes and was primarily aimed at searching for variations in EGFR and TP53 mutations. A comprehensive panel of genes may allow identification of specific gene mutations associated with osimertinib efficacy. The timing of the introduction of osimertinib varies from the second, third, and fourth to subsequent, and it is possible that the influence of drugs administered prior to the introduction of osimertinib caused bias in the genomic profile investigated to predict the therapeutic effect of osimertinib, yielding results different from those reported previously. The timing of image evaluation for the effects of osimertinib was also not defined in terms of parameters other than the sample collection; thus, the findings may not provide an accurate evaluation of osimertinib efficacy. Because the findings were not adjusted for the presence or absence of brain metastases and other distant metastases, systemic conditions at the start of treatment may have influenced osimertinib efficacy.

\section{Conclusion}

In this study, using serial plasma samples from patients harboring EGFR T790M and major activating mutations who were treated with osimertinib after second-line therapy, we analyzed the effect of osimertinib, the relationships among ctDNA biomarkers, and the changes in gene mutation profiles in ctDNA by utilizing the NOIR sequencing system. Multivariate analysis revealed that the fragment size of ctDNA with EGFR mutations could predict the effect of osimertinib. Since the size of ctDNA as well as the mutation profile can be used as biomarkers for predicting therapeutic effects, their use as predictors should be analyzed in a large-scale study in the future. 


\section{Methods}

All methods were carried out in accordance with relevant guidelines and regulations including declaration of Helsinki. The present study was a protocol-based retrospective study, and the study protocol (UMIN000028990) was approved by the ethics committees of each of the five participating institutes (Osaka International Cancer Institute, Osaka Toneyama Medical Center, Osaka Police Hospital, Osaka Habikino Medical Center and Osaka University Hospital). We enrolled NSCLC patients with biopsy-proven, advanced, nonsquamous NSCLC harboring EGFR T790M mutations in addition to the EGFR-activating major mutations exon (Ex.) 19 deletion (del) or Ex.21 L858R after EGFR-tyrosine kinase inhibitor (TKI) treatment based on the results of peptide nucleic acid-locked nucleic acid (PNA-LNA) real-time polymerase chain reaction(40); an Eastern Cooperative Oncology Group performance status of 0-2; and scheduled to receive oral osimertinib (at a dose of $80 \mathrm{mg}$ once daily). Treatment continued until disease progression, the development of unacceptable adverse effects, or a request by either the patient or physician to discontinue treatment. Patients with concurrent malignancy were ineligible, with the exception of those with non-melanoma skin cancer or noninvasive cervical cancer. Patients with a history of a prior cancer other than NSCLC were included if the previous diagnosis was performed more than 2 years prior to enrollment and the patient had no evidence of active disease. Patients had to have a life expectancy of at least 3 months. Patients with central nervous system metastases were eligible if they had been adequately treated and the neurological findings had returned to baseline. Patients with a history of osimertinib use were excluded from the study. Written informed consent was obtained from each patient or their guardian.

\section{Specimen collection and plasma isolation}

Blood samples $(20 \mathrm{~mL})$ were collected before starting osimertinib, one week and one month after starting osimertinib, and when resistance to osimertinib was recorded (Fig. 1A). Whole blood samples were collected in Streck cfDNA blood collection tubes, processed to plasma, and frozen within $96 \mathrm{~h}$. Whole blood was centrifuged at $1,600 \times g$ for $10 \mathrm{~min}$. The plasma supernatant was isolated and centrifuged twice at $4,122 \times g$ for 15 min (swinging bucket, break-off), aliquoted, and frozen at $-80^{\circ} \mathrm{C}$ for future use.

\section{Sample preparation, quality assessment, and NOIR-SS assay design}

Plasma preparation was performed as described previously(41). Cell-free DNA was extracted from patient plasma samples by using a QIAamp circulating nucleic acid kit (QIAGEN, Valencia, CA, USA). cfDNA was concentrated using Amicon $\mu$ ltra- 0.5 centrifugal filters. Double-stranded DNA was quantified using the Qubit dsDNA HS Assay (Thermo Fisher Scientific, Waltham, MA, USA) on a Qubit 2.0 Fluorometer (Thermo Fisher Scientific, Waltham, MA, USA). A molecular barcoded NGS library was constructed using the NOIR-SS method, as described previously(11). Purified cfDNA from $9 \mathrm{~mL}$ of blood was used as the starting material for the NOIR-SS assay. The amplicon panel (Table S1) covering lung cancer-related genes was used to amplify the target regions. The primers and adapters used for library construction are shown in Table S2. For each plasma sample, we prepared two reaction mixtures for forward and reverse gene-specific primer cocktails (Table S2) since our PCR system did not allow simultaneous use of 
forward and reverse primer pairs to avoid undesirable amplification between forward and reverse genespecific primers. The sequencing library was amplified by anchored PCR using a primer pair between a single anchor of a gene-specific primer and a universal primer on the sequencing adapter.

\section{Sequencing and data analysis}

The constructed library was quantified using the Qubit dsDNA HS Assay Kit or Quant-iT PicoGreen dsDNA Assay Kit (Thermo Fisher Scientific, Waltham, MA, USA) and loaded on an lon 540 chip using the lon Chef System (Thermo Fisher Scientific, Waltham, MA, USA). Sequencing was performed using the lon Torrent S5 XL platform. Data analysis was performed according to a previously described procedure(41). Reads in the FASTQ format were de-multiplexed using the 5-base indices for assignment of individuals. The sequences between the 5-base indices and spacer/linker sequences were obtained and used as barcode tags (unique molecular identifiers). Reads with more than $50 \mathrm{bp}$ were aligned to the target regions by using BWA-MEM(42), whereas reads with short mapped ends ( $<40$ bases) were discarded. Reads with the same molecular barcode sequences were grouped together, and erroneous barcode tags were detected and removed, as described previously. Consensus sequences for reads with the same barcode were created using VarScan(43) as described previously(11). When more than $85 \%$ of the reads had the same base at a position, this base was selected as the consensus base.

\section{Variant call by molecular barcoding analysis}

For variant detection, we applied a Poisson distribution model to calculate the sequencing error, as described previously(11). When the number of base alterations in a target region is significantly higher than the average expected from the sequencing error, the change may be attributable to variant(s). The sequencing error rate was set to 10-5, corresponding to the expectation of one alteration at a single nucleotide site by sequencing error in 100,000 analyzed DNA molecules. In this study, we evaluated each target site for the presence of variant(s), setting $\mathrm{P}=0.01$ as the threshold for variant detection. For hotspot mutations with variant-positive molecules, we evaluated each base position in the hotspot mutation at the specified threshold value estimated from anomaly detection(44). To remove artifactual mutations due to DNA damage or somatic mutations from normal hematopoietic stem cells (clonal hematopoiesis), CV78 variant filtering(41) was applied to select variants of somatic mutations by using the COSMIC database version 84. Common SNP sites and error-prone sites were excluded from the analysis. We used human genome build 37 (GRCh37/hg19) as the reference genome. The mutation variant was called from only a single mutant-positive DNA molecule when the probability of the observation estimated from the Poisson distribution under the null hypothesis was less than the $p$-value cutoff. The minimum \%VAF for the variant call depended on the total DNA fragments analyzed for the sample. On average, 3,589 cfDNA molecules were analyzed in the NOIR-SS assay in this study, corresponding to an average limit of detection \%VAF of $0.03 \%(1 / 3,589)$. Oncogenic mutations were defined as those appearing in addition to the main activating mutations, and mutations other than those registered as somatic mutations in COSMIC and common SNPs registered as HGVD were detected.

\section{ctDNA fragment size analysis}


Using the anchored PCR assay of NOIR-SS, the molecular tag adapter was attached to the blunt end terminal of cell-free DNA. The cfDNA from normal cells is considered to be fragmented by endogenous endonuclease digestion of nucleosome junctions at the time of apoptotic cell death. DNA damage generates short fragments of cfDNA during library preparation. Furthermore, cell death by neclosis or piroptosis causes disordered and random fragmentation, regardless of the nucleosome structure. Therefore, the fragment size pattern of cfDNA/ctDNA potentially contains information on the status of the tumor microenvironment, such as inflammation and the degree of abnormal cell death (necrosis/pyroptosis). The difference in cfDNA or ctDNA was discriminated according to the existence of a tumor marker sensitizing mutation (EGFR L858R, Ex.19 del, T790M) on the fragment DNA, and the fragment size distribution was estimated. The fragment size was estimated from the DNA length between the gene-specific primer-binding site and the terminal end of cfDNA. The $3 \sigma$ method $(45,46)$ was used to evaluate the fragment size of the EGFR-mutated ctDNA. Using this method, we defined "short fragment group" as those outside the standard range of ctDNA size.

\section{The EGFR allelic cis/trans location analysis}

The allelic cis/trans conformation of activating/resistance mutation pairs was assessed by target RNA sequencing using purified RNA as the starting material. First-strand cDNA was synthesized using Revertra-ace ${ }^{\circledR}$ (Toyobo), and multiplex PCR was performed using KOD Fx Neo (Toyobo). RT-PCR and sequence library construction were performed using the same assay conditions as described previously(47). Two sets of primer pairs to amplify activating/resistance mutation pairs were used to reveal the cis/trans configuration by analyzing the EGFR exon20-21 amplicon for T790M-L858R detection and the exon19-20 amplicon for Ex.19 del.-T790M detection. A total of $100 \mathrm{ng}$ of input RNA was used for each reverse transcription reaction assay. Sequence libraries for the lllumina platform from these PCR products were constructed using the GenNext ${ }^{\circledR}$ NGS Library Prep Kit (Toyobo). All procedures were performed according to the manufacturer's instructions. To determine the cis/trans configuration, approximately $5 \mathrm{~K}$ depth of reads in average was obtained for each amplicon and the minimum cutoff for mutation call was set to 10 positive reads per amplicon. The sample was judged as harboring compound mutations by cis conformation when the activating-resistance mutation pair was called on the single identical read, and it was otherwise judged as showing a trans conformation.

\section{Statistical analysis}

To analyze progression-free survival (PFS), times to events were estimated using the Kaplan-Meier method and compared using the log-rank test. Hazard ratios (HRs) for PFS were determined using a univariate Cox proportional hazards model. Cox proportional hazard models were used to evaluate several patient factors. To construct the multivariate model, we selected the most relevant PFS-related factors identified in the univariate analysis. All statistical analyses were performed using EZR software (Saitama Medical Center, Jichi Medical University, Saitama, Japan)(48). Statistical significance was set at $\mathrm{P}<0.05$. 


\section{Declarations}

Ethical approval: The present study was a protocol-based retrospective study, and the study protocol (UMIN000028990) was approved by the ethics committees of each participating institute. Informed consents were obtained from the all cases who were enrolled in the study.

\section{Data availability}

The datasets generated during and/or analyzed during the current study are available in the DDBJ Sequence Read Archive repository. DRA submission ID is PSUB016571.

\section{Author contributions}

K.K Fromal analysis, Investigation, Writing-original draft, K.N; Conceptualization, Investigation, Methodology, Y.S; Data curation, Investigation, Writing-original draft, M.M, S.I, H.S and I.N; Investigation,

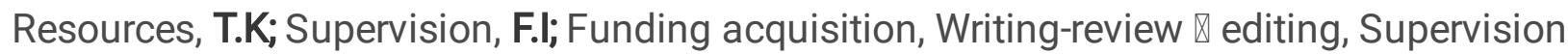

\section{Additional Information}

Disclosure Statement of Conflict of interest; This study supported by AstraZeneca fund. Dr. Kunimasa reports honoraria for lecture from AstraZeneca, Chugai Pharmaceutical and Novartis. Dr. Nishino reports a grant from Nippon Boehringer Ingelheim and honoraria for lecture from Chugai Pharmaceutical, AstraZeneca, Nippon Boehringer Ingelheim, Eli Lily Japan K.K, Roche Diagnostics, Novartis, Pfizer, Merk; Dr. Mori reports honoraria for lecture from AstraZeneca, MSD, Ono Pharmaceutical, Eli Lily Japan K.K, Boehringer Ingelheim, Novartis, Chugai Pharmaceutical, Taiho Pharmaceutical Co. Ltd, Kyowa-Kirin, Otsuka, Nihon-kayaku, Pfizer Japan Inc, Shionogi; Dr. Suzuki reports honoraria for lecture from AstraZeneca, Ono Pharmaceutical, Chugai Pharmaceutical, MSD, Bristol-Myers Squibb, Eli Lily Japan K.K; Dr. Nagatomo reports honoraria for lecture from AstraZeneca, Nippon Boehringer Ingelheim, Nippon Kayaku Co., Ltd, Eli Lily Japan K.K.; Dr. Kumagai reports grants from Ono Pharmaceutical, MSD, Chugai Pharceutical Co. Ltd, AstraZeneca, Takeda Pharmaceutical Companey Limited. Regeneron Pharmaceuticals, Inc. Merck Serono Co., Ltd. Pfizer Japan Inc. Taiho Pharmaceutical Co.,Ltd. Nippon Boehringer Ingelheim Co., Ltd. Eli Lilly Japan. Novartis Pharma. AbbVie GK., Delta-Fly Pharma, Inc. The Osaka Foundation for The Prevention of Cancer and Life-style related Diseases (Public Interest Incorporated Foundation), and honoraria from Ono Pharmaceutical, AstraZeneca, Taiho Pharmaceutical Co. Ltd., MSD, TEIJIN PHARMA LIMITED, Novartis Pharma, Nippon Boehringer Ingelheim Co., Ltd. Eli Lily Japan. Pfizer Inc., Chugai Pharceutical Co. Ltd., Bristol-Myers Squibb; Dr. Imamura reports honoraria for lecture from AstraZeneca, Chugai Pharma and Nippon Boehringer Ingelheim, and other authors have no conflict of interest.

\section{References}


1. Planchard D, et al. Metastatic non-small cell lung cancer: ESMO Clinical Practice Guidelines for diagnosis, treatment and follow-up. Ann Oncol 2018;29:iv192-iv237.

2. Ettinger DS, et al. NCCN Guidelines Insights: Non-Small Cell Lung Cancer, Version 1.2020. J Natl Compr Canc Netw 2019;17:1464-1472.

3. Aggarwal $\mathrm{C}$, et al. Strategies for the successful implementation of plasma-based NSCLC genotyping in clinical practice. Nat Rev Clin Oncol 2021;18:56-62.

4. Kunimasa $\mathrm{K}$, et al. Improvement strategies for successful next-generation sequencing analysis of lung cancer. Future Oncol 2020;16:1597-1606.

5. Leighl NB, et al. Clinical Utility of Comprehensive Cell-free DNA Analysis to Identify Genomic Biomarkers in Patients with Newly Diagnosed Metastatic Non-small Cell Lung Cancer. Clin Cancer Res 2019;25:4691-4700.

6. Saarenheimo J, Andersen H, Eigeliene N, Jekunen A. Gene-Guided Treatment Decision-Making in Non-Small Cell Lung Cancer - A Systematic Review. Front Oncol 2021;11:754427.

7. Rolfo $C$, et al. Liquid Biopsy for Advanced NSCLC: A Consensus Statement From the International Association for the Study of Lung Cancer. J Thorac Oncol 2021;16:1647-1662.

8. Laufer-Geva S, et al. The Clinical Impact of Comprehensive Genomic Testing of Circulating Cell-Free DNA in Advanced Lung Cancer. J Thorac Oncol 2018;13:1705-1716.

9. Woodhouse R, et al. Clinical and analytical validation of FoundationOne Liquid CDx, a novel 324Gene cfDNA-based comprehensive genomic profiling assay for cancers of solid tumor origin. PLoS One 2020;15:e0237802.

10. Newman AM, et al. An ultrasensitive method for quantitating circulating tumor DNA with broad patient coverage. Nat Med 2014;20:548-54.

11. Kukita Y, et al. High-fidelity target sequencing of individual molecules identified using barcode sequences: de novo detection and absolute quantitation of mutations in plasma cell-free DNA from cancer patients. DNA Res 2015;22:269-77.

12. Casbon JA, Osborne RJ, Brenner S, Lichtenstein CP. A method for counting PCR template molecules with application to next-generation sequencing. Nucleic Acids Res 2011;39:e81.

13. Kinde I, Wu J, Papadopoulos N, Kinzler KW, Vogelstein B. Detection and quantification of rare mutations with massively parallel sequencing. Proc Natl Acad Sci U S A 2011;108:9530-5.

14. Akahori D, et al. Comparative assessment of NOIR-SS and ddPCR for ctDNA detection of EGFR L858R mutations in advanced L858R-positive lung adenocarcinomas. Sci Rep 2021;11:14999.

15. Takada R, et al. Clinical Utility of Pancreatic Cancer Circulating Tumor DNA in Predicting Disease Progression, Prognosis, and Response to Chemotherapy. Pancreas 2020;49:e93-e95.

16. Park S, et al. High concordance of actionable genomic alterations identified between circulating tumor DNA-based and tissue-based next-generation sequencing testing in advanced non-small cell lung cancer: The Korean Lung Liquid Versus Invasive Biopsy Program. Cancer 2021;127:3019-3028. 
17. Leon SA, Shapiro B, Sklaroff DM, Yaros MJ. Free DNA in the serum of cancer patients and the effect of therapy. Cancer Res 1977;37:646-50.

18. Mouliere $F$, et al. High fragmentation characterizes tumour-derived circulating DNA. PLoS One 2011;6:e23418.

19. Yamamoto $Y$, et al. Clinical significance of the mutational landscape and fragmentation of circulating tumor DNA in renal cell carcinoma. Cancer Sci 2019;110:617-628.

20. Liu X, et al. Enrichment of short mutant cell-free DNA fragments enhanced detection of pancreatic cancer. EBioMedicine 2019;41:345-356.

21. Underhill HR, et al. Fragment Length of Circulating Tumor DNA. PLoS Genet 2016;12:e1006162.

22. Liu $Y$, et al. Increased detection of circulating tumor DNA by short fragment enrichment. Transl Lung Cancer Res 2021;10:1501-1511.

23. Mouliere F, et al. Enhanced detection of circulating tumor DNA by fragment size analysis. Sci Transl Med 2018;10.

24. Underhill HR. Leveraging the Fragment Length of Circulating Tumour DNA to Improve Molecular Profiling of Solid Tumour Malignancies with Next-Generation Sequencing: A Pathway to Advanced Non-invasive Diagnostics in Precision Oncology? Mol Diagn Ther 2021;25:389-408.

25. Li F, et al. Ultra-Short Circulating Tumor DNA (usctDNA) in Plasma and Saliva of Non-Small Cell Lung Cancer (NSCLC) Patients. Cancers (Basel) 2020;12.

26. Heitzer E, Auinger L, Speicher MR. Cell-Free DNA and Apoptosis: How Dead Cells Inform About the Living. Trends Mol Med 2020;26:519-528.

27. Jahr S, et al. DNA fragments in the blood plasma of cancer patients: quantitations and evidence for their origin from apoptotic and necrotic cells. Cancer Res 2001;61:1659-65.

28. Jensen TJ, et al. Whole genome bisulfite sequencing of cell-free DNA and its cellular contributors uncovers placenta hypomethylated domains. Genome Biol 2015;16:78.

29. Udomruk S, Orrapin S, Pruksakorn D, Chaiyawat P. Size distribution of cell-free DNA in oncology. Crit Rev Oncol Hematol 2021;166:103455.

30. Ying HQ, et al. Cancer-elicited inflammation attenuates response and outcome in tyrosine kinase inhibitor naive patients with advanced NSCLC. Pharmacol Res 2021;170:105734.

31. Chen YM, et al. Impact of clinical parameters and systemic inflammatory status on epidermal growth factor receptor-mutant non-small cell lung cancer patients readministration with epidermal growth factor receptor tyrosine kinase inhibitors. BMC Cancer 2016;16:868.

32. Garrido P, et al. LungBEAM: A prospective multicenter study to monitor stage IV NSCLC patients with EGFR mutations using BEAMing technology. Cancer Med 2021;10:5878-5888.

33. Sueoka-Aragane $\mathrm{N}$, et al. The role of comprehensive analysis with circulating tumor DNA in advanced non-small cell lung cancer patients considered for osimertinib treatment. Cancer Med 2021;10:38733885 . 
34. Sakai K, et al. Predicting osimertinib-treatment outcomes through EGFR mutant-fraction monitoring in the circulating tumor DNA of EGFR T790M-positive patients with non-small cell lung cancer (WJOG8815L). Mol Oncol 2021;15:126-137.

35. Mok TS, et al. Osimertinib or Platinum-Pemetrexed in EGFR T790M-Positive Lung Cancer. N Engl J Med 2017;376:629-640.

36. Papadimitrakopoulou VA, et al. Osimertinib versus platinum-pemetrexed for patients with EGFR T790M advanced NSCLC and progression on a prior EGFR-tyrosine kinase inhibitor: AURA3 overall survival analysis. Ann Oncol 2020;31:1536-1544.

37. Oxnard GR, et al. Assessment of Resistance Mechanisms and Clinical Implications in Patients With EGFR T790M-Positive Lung Cancer and Acquired Resistance to Osimertinib. JAMA Oncol 2018;4:1527-1534.

38. Wang F, et al. Genomic temporal heterogeneity of circulating tumour DNA in unresectable metastatic colorectal cancer under first-line treatment. Gut 2021.

39. Mouliere F, et al. Fragmentation patterns and personalized sequencing of cell-free DNA in urine and plasma of glioma patients. EMBO Mol Med 2021;13:e12881.

40. Nagai Y, et al. Genetic heterogeneity of the epidermal growth factor receptor in non-small cell lung cancer cell lines revealed by a rapid and sensitive detection system, the peptide nucleic acid-locked nucleic acid PCR clamp. Cancer Res 2005;65:7276-82.

41. Kukita Y, et al. Selective identification of somatic mutations in pancreatic cancer cells through a combination of next-generation sequencing of plasma DNA using molecular barcodes and a bioinformatic variant filter. PLoS One 2018;13:e0192611.

42. Li H, Durbin R. Fast and accurate short read alignment with Burrows-Wheeler transform. Bioinformatics 2009;25:1754-60.

43. Koboldt DC, et al. VarScan 2: somatic mutation and copy number alteration discovery in cancer by exome sequencing. Genome Res 2012;22:568-76.

44. Kukita Y, et al. Quantitative identification of mutant alleles derived from lung cancer in plasma cellfree DNA via anomaly detection using deep sequencing data. PLoS One 2013;8:e81468.

45. Cao Y, Xie L, Xie Y, Xu H. Sequential Change-Point Detection via Online Convex Optimization. Entropy (Basel, Switzerland) 2018;20.

46. Liu S, Yamada M, Collier N, Sugiyama M. Change-point detection in time-series data by relative density-ratio estimation. Neural Netw 2013;43:72-83.

47. Kato K, et al. Analytical performance of a highly sensitive system to detect gene variants using nextgeneration sequencing for lung cancer companion diagnostics. medRxiv 2021:2021.10.13.21264976.

48. Kanda Y. Investigation of the freely available easy-to-use software 'EZR' for medical statistics. Bone Marrow Transplant 2013;48:452-8. 


\section{Tables}

Table.1 Patients' characteristics

\begin{tabular}{llcc}
\hline & & $\begin{array}{c}\text { Enrolled patients } \\
\text { (n=34) }\end{array}$ & (\%) \\
\hline Age-median, [range] & Median & $66[39-83]$ & \\
Sex-no. (\%) & Male & 7 & $(20.6)$ \\
Smoking & Female & 27 & $(79.4)$ \\
& Never & 22 & $(64.7)$ \\
PS & Ex- or Current & 12 & $(35.3)$ \\
& 0 & 14 & $(41.2)$ \\
Histology-no. (\%) & 1 & 16 & $(47.1)$ \\
& 2 & 4 & $(11.7)$ \\
Stage-n. (\%) & Adenocarcinoma & 33 & $(97.1)$ \\
& Adenosquamous & 1 & $(2.9)$ \\
Brain metastases at osimertinib & 7 & $(20.6)$ \\
EGFR mutation & IVA & 27 & $(79.4)$ \\
& & 17 & $(50.0)$ \\
Osimertinib treatment line & Ex. 19 deletion & 20 & $(58.8)$ \\
& 2 & 14 & $(41.2)$ \\
& 3 & 10 & $(29.4)$ \\
& $4 \geqq$ & 11 & $(32.4)$ \\
& & 13 & $(38.2)$ \\
\hline
\end{tabular}

Table.2 Analysis of clinical and ctDNA risk factors for PFS of osimertinib

\begin{tabular}{|c|c|c|c|c|c|c|}
\hline \multirow[b]{2}{*}{ Variables } & & \multirow[b]{2}{*}{$(\%)$} & \multicolumn{2}{|c|}{ Univariate } & \multicolumn{2}{|c|}{ Multivariate } \\
\hline & & & ORR $(95 \% \mathrm{Cl})$ & $\mathrm{P}$ & ORR $(95 \% \mathrm{Cl})$ & $\mathrm{P}$ \\
\hline$\overline{\text { EGFR major mutations }}$ & Ex.19 del & $(58.8)$ & 1 & reference & 1 & reference \\
\hline & L858R & $(41.2)$ & $1.60(0.77-3.33)$ & 0.209 & $1.65(0.68-4.00)$ & 0.266 \\
\hline EGFR-mutated ctDNA fragment & $\begin{array}{l}\text { long } \\
\text { short }\end{array}$ & $\begin{array}{l}(76.5) \\
(23.5)\end{array}$ & $\begin{array}{c}1 \\
4.08(1.54-10.8)\end{array}$ & $\begin{array}{l}\text { reference } \\
<0.05\end{array}$ & $\begin{array}{c}1 \\
3.76(1.17-12.1)\end{array}$ & $\begin{array}{c}\text { reference } \\
<0.05\end{array}$ \\
\hline Disappearance timing & $\begin{array}{l}\mathrm{A} \rightarrow \mathrm{B} \text { disappear } \\
\text { Others }\end{array}$ & $\begin{array}{l}(26.5) \\
(735)\end{array}$ & $\begin{array}{c}1 \\
168(0.75-377)\end{array}$ & $\begin{array}{c}\text { reference } \\
0.21\end{array}$ & $\begin{array}{c}1 \\
213(0.79-5.72)\end{array}$ & $\begin{array}{c}\text { reference } \\
0.135\end{array}$ \\
\hline Clonal evolution patterns & $\begin{array}{l}\text { Additional pattern } \\
\text { Others }\end{array}$ & $\begin{array}{l}(17.6) \\
(82.4)\end{array}$ & $\begin{array}{c}1 \\
3.29(1.81-15.5)\end{array}$ & $\begin{array}{c}\text { reference } \\
<0.05\end{array}$ & $\begin{array}{c}1 \\
1.16(0.74-1.82)\end{array}$ & $\begin{array}{c}\text { reference } \\
0.517\end{array}$ \\
\hline Co-mutations & $\begin{array}{l}(-) \\
(+)\end{array}$ & $\begin{array}{l}(29.4) \\
(70.6) \\
\end{array}$ & $\begin{array}{c}1 \\
1.07(0.50-2.29) \\
\end{array}$ & $\begin{array}{c}\text { reference } \\
0.869 \\
\end{array}$ & $\begin{array}{c}1 \\
0.90(0.35-2.26) \\
\end{array}$ & $\begin{array}{c}\text { reference } \\
0.815 \\
\end{array}$ \\
\hline
\end{tabular}

Figures

Figure 1

(A) Schematic representation of the research protocol. Peripheral blood was collected before administration of osimertinib (Point A), one week after administration (Point B), one month after 
administration (Point C), and when osimertinib resistance was identified (Point D). (B) Violin plot of EGFR wild-type and mutated ctDNA fragmentations. White circles ( $(\mathrm{C})$ indicate median levels. The plots represent individual EGFR-mutated alleles and the respective number of cases; ${ }^{*} p<0.05$. (C) The median, standard deviation (SD), and cut-off values of each fragment size of EGFR T790M, L858R, and Ex.19 deletion alleles. (D) Kaplan-Meier plot of PFS for osimertinib treatment. Comparison of PFS for EGFR-mutated patients with the shorter EGFR-mutated allele fraction (red line) vs. others (black line); HR for the long group: $4.08(95 \% \mathrm{Cl}, 1.54-10.8 ; \mathrm{p}<0.05)$.

\section{Figure 2}

(A) A scatterplot graph and correlation analysis $(r=0.837)$ results for VAF of the EGFR Ex.19 deletion or L858R mutations ( $\mathrm{X}$ axis) and the EGFR T790M mutations ( $\mathrm{Y}$ axis) (B) The cis-trans configuration between the activating and resistance mutations was analyzed by target RNA-Seq analysis of tissue biopsy specimens. This figure shows one example of a cis pattern of Ex.19 deletion and T790M visualized by the IGV genome browser. The region bridging Ex.19 and Ex.20 of EGFR was amplified by RTPCR and the amplicon was sequenced by NGS. Most of sequencing reads with a 15-bp Ex.19 deletion (represented by the blue line and number) harbor T790M mutations on the same RNA molecule. (C) Comparison of the VAF of EGFR T790M at Point A between the no disappearance, $A \rightarrow B$ disappearance, and $A \rightarrow C$ disappearance groups. NS; no significance. (D) Kaplan-Meier plot of PFS for osimertinib treatment. Comparison of PFS between the no disappearance, $A \rightarrow B$ disappearance, and $A \rightarrow C$ disappearance groups $(p=0.442)$.

\section{Figure 3}

(A) Representative heat maps of clonal evolution types (new driver mutation type, additional mutation type, and attenuation type). The $Y$ axis represents each mutation, and the $X$ axis represents VAF from Point $A$ to $D$. The percentage of VAF is expressed in alongside heat map tones. In the new driver mutation type, EGFR mutation disappears and a new driver mutation appears in Point D. In the additional mutation type, additional driver mutations appear in addition to EGFR mutation. In the attenuation pattern, driver mutation only of EGFR mutation is confirmed even in Point D. (B) Kaplan-Meier plot of PFS for osimertinib treatment. Comparison of PFS between the new driver mutation type, additional mutation type, and attenuation type $(p<0.05)$.

\section{Figure 4}


(A) Oncogene mutation concordance chart for mutated genes across patients analyzed by tissue-liquidmatched pairs. A total of 19 patients were tested using both NOIR-SS NGS assays. The detected mutations were colored according to the concordance status. (B) Mutation fractions of the shared versus tissue- or liquid-only mutations. (C) Heatmap of ctDNA mutations detected by liquid biopsy of Point A. The detected mutations were colored based on their $\log _{10}$ VAF values. The mutation with the largest VAF value was selected in this heatmap when multiple mutations were detected for TP53. (D) Kaplan-Meier plot of PFS for osimertinib treatment. Comparison of PFS between co-mutation(-) and co-mutation(+) groups. $\mathrm{p}$-value $=0.749$.

\section{Supplementary Files}

This is a list of supplementary files associated with this preprint. Click to download.

- SupplementaryTables.xlsx 